\title{
Coil embolization of ruptured intrahepatic pseudoaneurysm through percutaneous transhepatic biliary drainage
}

\author{
Jee Young $\mathrm{An}^{1}$, Jae Sin Lee1, Dong Ryul Kim¹, Jae Young Jang${ }^{1}$, Hwa Young Jung ${ }^{1}$, \\ Jong Ho Park ${ }^{2}$, Sue Sin $\mathrm{Jin}^{2}$ \\ ${ }^{I}$ Department of Internal Medicine, College of Medicine, The Catholic University of Korea, Seoul; \\ ${ }^{2}$ Department of Internal Medicine, Changwon Fatima Hospital, Changwon, Korea
}

\begin{abstract}
A 75-year-old man with chronic cholangitis and a common bile duct stone that was not previously identified was admitted for right upper quadrant pain. Acute cholecystitis with cholangitis was suspected on abdominal computed tomography (CT); therefore, endoscopic retrograde cholangiopancreatography with endonasal biliary drainage was performed. On admission day 5, hemobilia with rupture of two intrahepatic artery pseudoaneurysms was observed on follow-up abdominal CT. Coil embolization of the pseudoaneurysms was conducted using percutaneous transhepatic biliary drainage. After several days, intrahepatic artery pseudoaneurysm rupture recurred and coil embolization through a percutaneous transhepatic biliary drainage tract was conducted after failure of embolization via the hepatic artery due to previous coiling. After the second coil embolization, a common bile duct stone was removed, and the patient presented no complications during 4 months of follow-up. We report a case of intrahepatic artery pseudoaneurysm rupture without prior history of intervention involving the hepatobiliary system that was successfully managed using coil embolization through percutaneous transhepatic biliary drainage.
\end{abstract}

Keywords: Hepatic artery; Pseudoaneurysm; Hemobilia; Embolization

\section{INTRODUCTION}

A hepatic artery aneurysm (HAA) is very rare, with an estimated prevalence of $0.002 \%$. Most HAA patients are asymptomatic until the aneurysm ruptures. However, ruptures occur in $21-80 \%$ of cases and can result in a mortality rate of 21-43\% [1-3]. Depending on the composition of the wall, aneurysms may be divided into true aneurysms or pseudoaneurysms. The incidence of hepatic artery pseudoaneurysms has

Received: August 17, 2017, Revised: October 18, 2017 Accepted: October 19, 2017

Corresponding Author: Jong Ho Park, Department of Internal Medicine, Changwon Fatima hospital, 45, Changi-daero, Uichang-gu, Changwon 51394, Korea Tel: +82-2-55-270-1000, Fax: +82-2-55-265-7766

E-mail: 4796pjhsms@naver.com been increasing due to complications following surgery or procedures involving the hepatobiliary system [1].

The authors report a case of ruptured intrahepatic pseudoaneurysm and rebleeding treated by embolization through a percutaneous transhepatic biliary drainage (PTBD) catheter in a patient with no history of surgery or procedures involving the hepatobiliary system.

\section{CASE}

A 75-year-old male was emergently admitted for the chief complaint of right upper quadrant (RUQ) pain and fever for 3 days. The patient had no underlying diseases, medication history, including anticoagulant use, or history of surgery or abdominal procedures. Physical examination showed blood pressure (BP) 105/60 mmHg, pulse 98 beats/min, respirations

Copyright (C) 2018 Yeungnam University College of Medicine

This is an Open Access article distributed under the terms of the Creative Commons Attribution Non-Commercial License (http://creativecommons.org/licenses/by-nc/4.0/) which permits unrestricted non-commercial use, distribution, and reproduction in any medium, provided the original work is properly cited. 
$16 /$ min, and body temperature $37.8^{\circ} \mathrm{C}$. The patient appeared acutely ill, with tenderness in the RUQ, but no abdominal rigidity. The white blood cell count was $9,930 / \mathrm{mm}^{3}$, with hemoglobin $(\mathrm{Hb}) 9.6 \mathrm{~g} / \mathrm{dL}$, platelet count $363,000 / \mathrm{mm}^{3}$, total bilirubin $4.7 \mathrm{mg} / \mathrm{dL}$, direct bilirubin $3.8 \mathrm{mg} / \mathrm{dL}$, aspartate transaminase (AST) $315 \mathrm{IU} / \mathrm{L}$, alanine transaminase (ALT) 114 IU/L, alkaline phosphatase (ALP) 370 IU/L, and prothrombin time $14.6 \mathrm{~s}$ (international normalized ratio 1.31).
Abdominal computed tomography (CT) performed on the day of admission showed intra-gallbladder hematoma and findings of partial expansion of the intrahepatic bile duct caused by hemobilia (Fig. 1). Upper gastrointestinal endoscopy showed edema in the duodenal ampulla of Vater, but no bleeding. The patient was diagnosed with acute cholangitis, and his progress was monitored with antibiotic maintenance after endoscopic nasobiliary drainage.
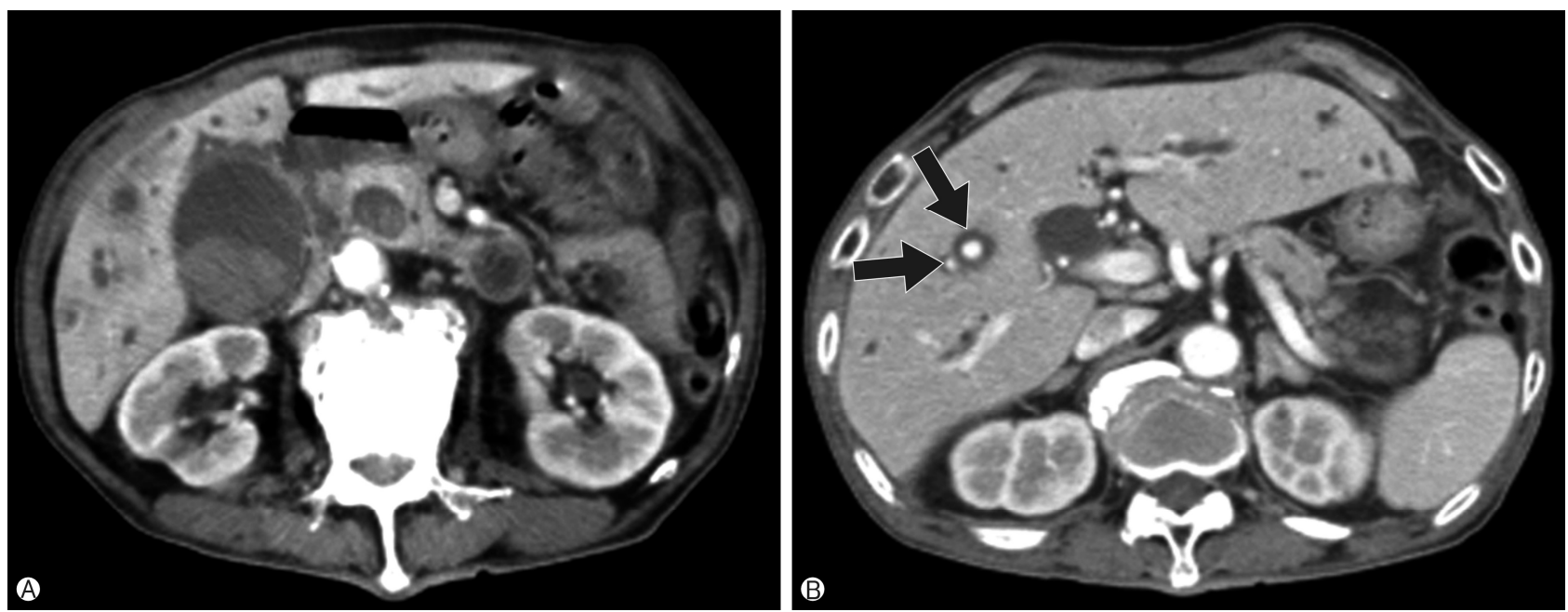

Fig. 1. Initial abdominal computed tomography. (A) High density materials are seen in gallbladder and common bile duct with marked biliary dilatation and pericholecystic infiltrations suggesting intramural gallbladder hematoma and cholangitis. (B) Suspcious intrahepatic aneurysms (arrows) are seen at liver segment 5 and hemobilia induced biliary dilatations are observed.
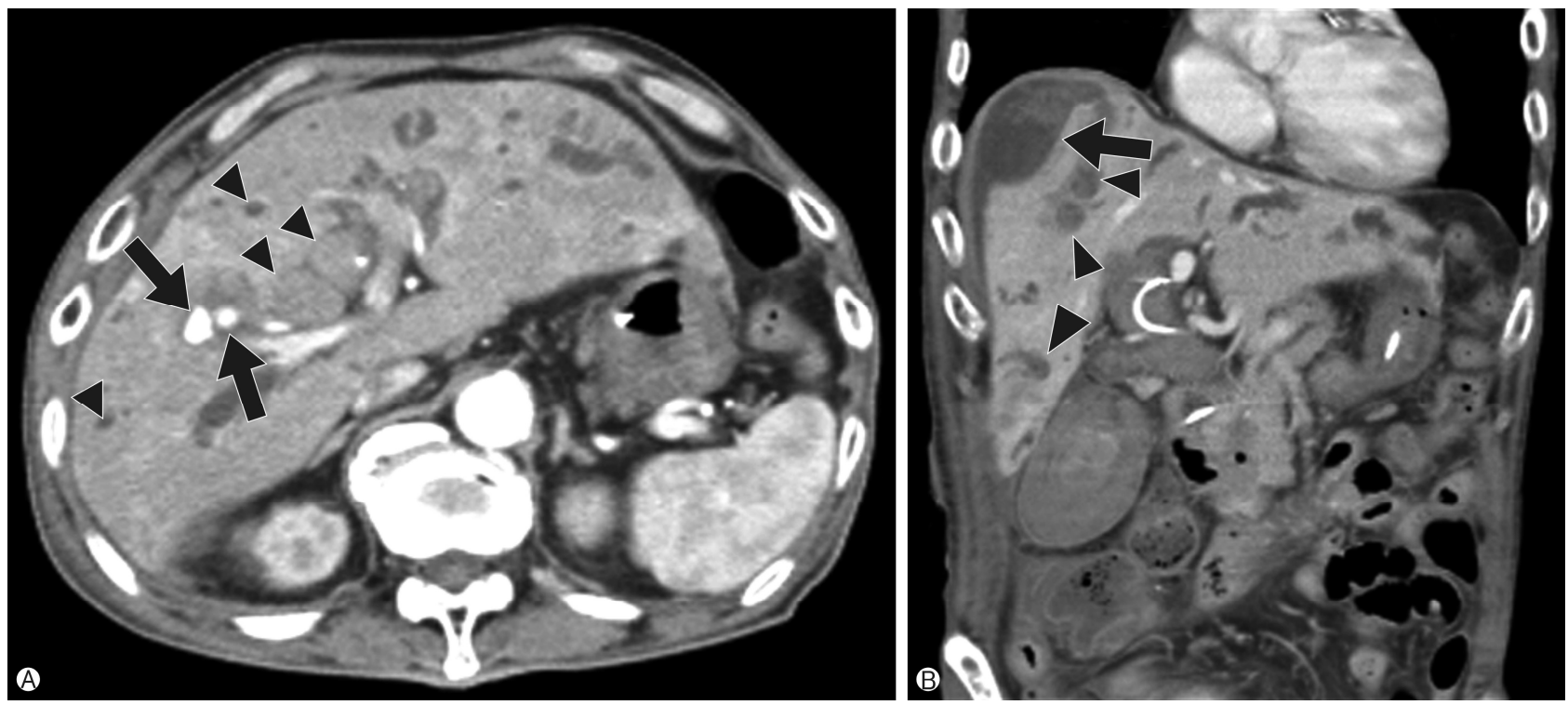

Fig. 2. Follow-up abdominal computed tomography. (A) Two intrahepatic psuedoaneurysms (arrows) with partial rupture and active bleeding at liver segment 5 is observed with intrabiliary hematoma and biliary dilatation (arrow heads). (B) Intrahepatic bile duct dilatation (arrow head) and right perihepatic subcapsular hematoma (arrow) at liver dome due to rupture and spillage of intrahepatic bile duct are observed. 
On the following day, RUQ pain persisted; percutaneous transhepatic gallbladder drainage (PTGBD) was performed and the fluid was bloody. On the 5th hospital day, the RUQ pain worsened. While BP $(110 / 70 \mathrm{mmHg})$ and pulse $(75$ beats $/ \mathrm{min})$ remained stable, $\mathrm{Hb}$ decreased from $8.8 \mathrm{~g} / \mathrm{dL}$ to $7.3 \mathrm{~g} / \mathrm{dL}$, and total bilirubin increased to $5.2 \mathrm{mg} / \mathrm{dL}$, with AST $54 \mathrm{IU} / \mathrm{L}$, ALT $20 \mathrm{IU} / \mathrm{L}$, and ALP $128 \mathrm{IU} / \mathrm{L}$. Abdominal CT showed hemobilia and two intrahepatic pseudoaneurysms located in the fifth liver segment (Fig. 2). Emergency hepatic arterial angiography was performed; after confirming a ruptured intrahepatic pseudoaneurysm (Fig. 3A), coil embolization and PTBD were performed for hemostasis and biliary drainage (Fig. 3B). The patient stabilized, but on the 15th hospital day, the RUQ pain recurred. His BP and pulse were stable, but $\mathrm{Hb}$ decreased slightly from $9.0 \mathrm{~g} / \mathrm{dL}$ to $8.3 \mathrm{~g} / \mathrm{dL}$ and total bilirubin increased to $9.2 \mathrm{mg} / \mathrm{dL}$. Abdominal CT was repeated. Hemobilia and coil-embolized intrahepatic pseudoaneurysms were enhanced. Emergency hepatic arterial angiography was performed for possible recurrent rupture of the intrahepatic pseudoaneurysms (Fig. 4A). Although another transarterial coil embolization was planned, superselection was impossible due to a previously inserted coil and winding vascular path. However, a microcatheter was able to approach the biliary-intrahepatic pseudoaneurysm fistula through the PTBD catheter that had been inserted (Fig. 4B), and coil embolization of the intrahepatic pseudoaneurysm was successfully completed (Fig. 4C).

Subsequently, clinical improvement continued and PTGBD and PTBD catheters were removed. When removing the PTBD catheter, bile duct stones were also removed. The patient was discharged after 35 days of hospitalization and followed up

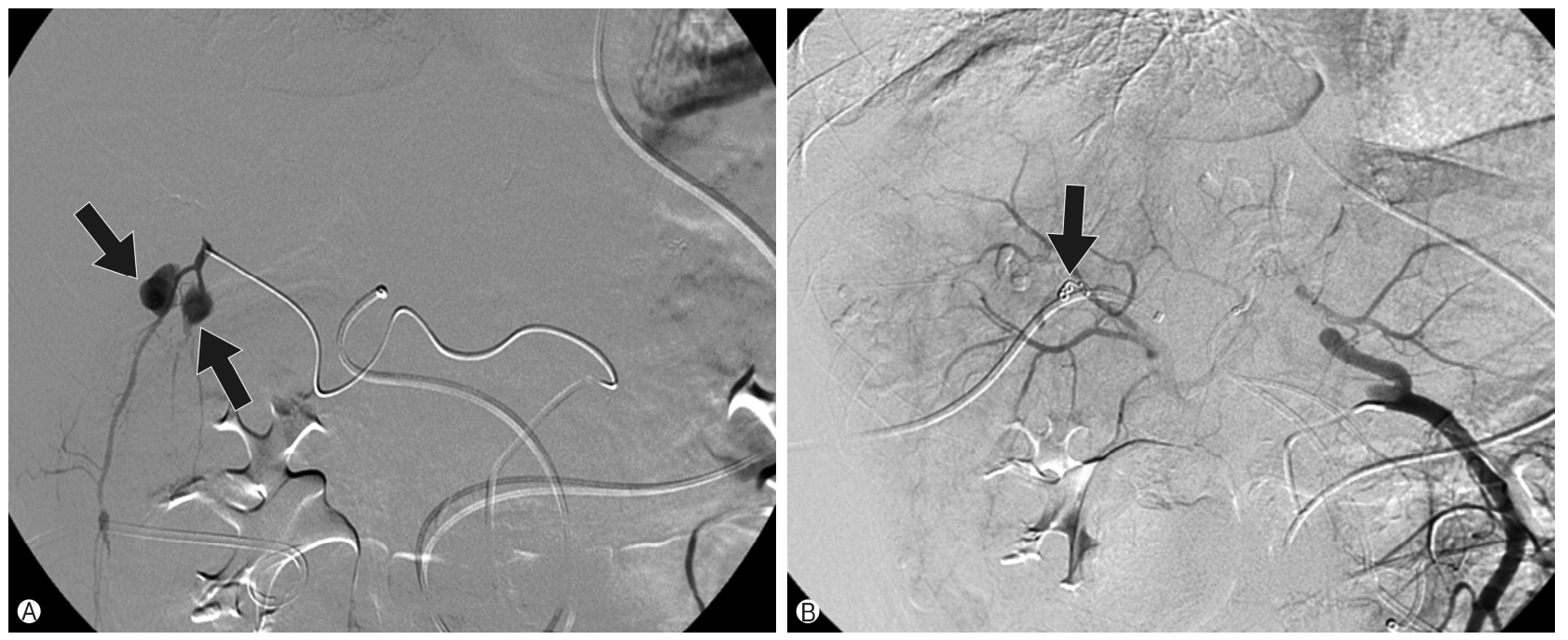

Fig. 3. Initial hepatic artery angiography. (A) Two intrahepatic pseudoaneurysms (arrows) at liver segment 5 are seen. (B) Inserted coils (arrow) for embolization of the intrahepatic pseudoaneurysm are observed.
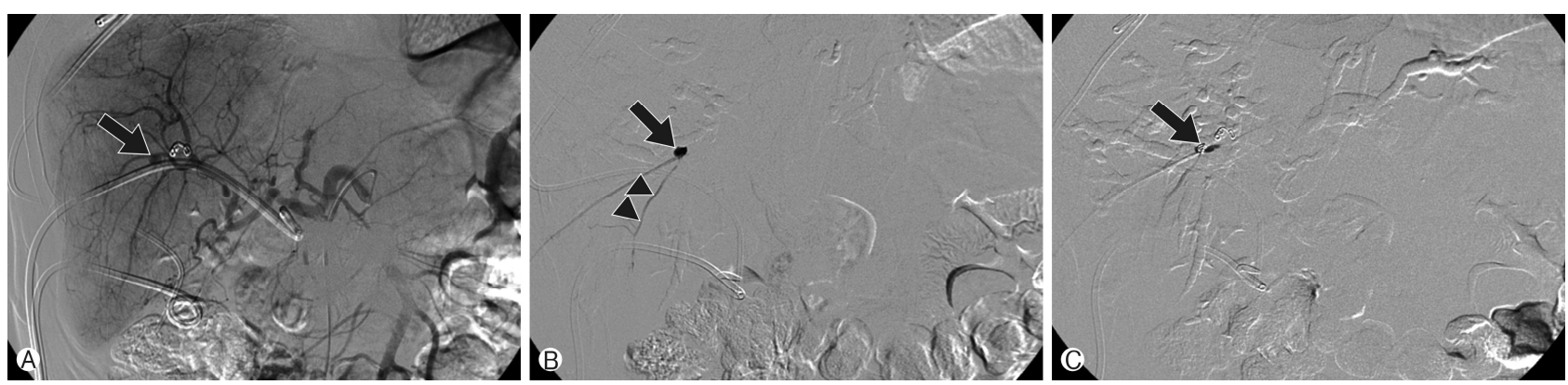

Fig. 4. Follow-up hepatic artery angiography. (A) Recurred intrahepatic pseudoaneurysm (arrow) is observed at liver segment 5. (B) After approach of the intrahepatic bile duct - intrahepatic pseudoaneursym fistula using microcatheter through a percutaneous transhepatic biliary drainage tract, the psuedoaneurysm (arrow) and intrahepatic artery (arrow head) are seen. (C) Inserted coils (arrow) for embolization of recurred intrahepatic pseudoaneurysm are observed. 
for 4 months, during which time there were no signs of recurrence.

\section{DISCUSSION}

HAA is very rare. However, among intra-abdominal aneurysms, it has the highest prevalence after splenic aneurysms, accounting for 20-40\% [1-4].

True aneurysms are mostly found as single, extrahepatic lesions, whereas pseudoaneurysms are mostly found as multiple, intrahepatic lesions. Pseudoaneurysms often occur as a result of complications from surgeries and procedures that involve the biliary tract, in addition to abdominal trauma. However, in rare cases, they may also be caused by inflammation around the artery due to pancreatitis. In the past, true aneurysms were more common, but with a recent increase in surgeries and procedures that involve the biliary tract, the occurrence of pseudoaneurysms is increasing, although the exact prevalence is difficult to measure [1,5-7]. A study by Tessier et al. analyzed the medical records of 153 patients with hepatic artery pseudoaneurysms and found that occurrence after PTBD was most common, accounting for 31\%, followed in order by laparoscopic cholecystectomy (28\%), open cholecystectomy (13\%), and percutaneous liver biopsy (7\%) [7]. The risk factors associated with iatrogenic pseudoaneurysms include a history of anticoagulant use, female sex, obesity, and vascular calcification [11]. The present case involved natural rupture of intrahepatic pseudoaneurysms in a patient with chronic cholangitis caused by bile duct stones. Based on review of other reports, the cause of intrahepatic pseudoaneurysms in the present case can be considered as being very rare.

Approximately $60 \%$ of HAA patients are symptomatic, with the most common symptoms being epigastric and RUQ pain, while Quincke's triad (abdominal pain, jaundice, and hemobilia) appear in only about $33 \%$ of patients $[2,8]$. In the present case, hemobilia was present in the fluid drained via PTGBD performed on the day after admission; thus, Quincke's triad was fulfilled. HAA may rupture in the hepatic vein, portal vein, bile duct, or directly into the abdominal cavity, and is diagnosed for the first time in $20-80 \%$ of patients when rupture occurs [2,6,9]. Mortality associated with HAA rupture is reportedly $21-43 \%$, while the risk of rupture is high when the aneurysm is $\geq 2 \mathrm{~cm}[2,9]$.
Abdominal sonography is used for diagnostic screening of HAA, but in most cases, abdominal CT is performed to confirm the relationship between the size of the HAA and surrounding structures [1,6]. Selective angiography is the most sensitive and specific method for diagnosing HAA, and provides important pre- and intra-operative information, such as the location of the aneurysm, anatomical anomalies, and collateral circulation $[2,3,6]$.

In the past, most authors recommended that HAA should be treated regardless of the symptoms due to the risk of rupture and death. Recently, however, not all asymptomatic HAA cases are treated. For asymptomatic atherosclerotic HAA, treatment is recommended only when the diameter is $\geq 2 \mathrm{~cm}$, but a case involving non-atherosclerotic multiple aneurysms must be treated regardless of the size due to the high risk of symptoms and rupture [1,9]. Treatment methods may vary according to the location and shape of HAA, as well as the patient's condition $[3,7,8]$. For extrahepatic artery aneurysms, embolization may be an option if the aneurysm sac has a pocket shape and adequate hard area. However, for extrahepatic artery aneurysms with a spindle shape or lacking a hard area, surgery should be considered [3,8]. In the past, intrahepatic artery aneurysms required liver resection, but since hepatic artery embolization was introduced in 1977, embolization is performed on most patients, using Gelfoam, coils, and spirals [8]. The success rate of hepatic artery embolization is reportedly $88-100 \%$ [6]. Embolization has the advantage of minimizing hepatic parenchymal ischemia, but may also cause occlusion in normal blood vessels or liver failure due to backflow of the material used in embolization. Moreover, re-embolization may also be required since recurrence is found in approximately $37-42 \%$ of patients $[6-8,10]$. If embolization cannot be performed through the hepatic artery or rupture of the intrahepatic artery aneurysm continues despite hepatic artery embolization, ultrasound-guided percutaneous embolization may be performed by directly puncturing the aneurysm sac [2,3]. Surgery involves removal of the aneurysm and suturing the arterial wall or applying a patch suture, with the advantages of reducing the incidence of rebleeding, as well as reducing embolization-related complications, such as vascular occlusion and liver failure [11]. However, data on comparisons between embolization and surgery showed that both have similar efficacy, but embolization is preferred since it reduces the duration of hospital stay and the need for transfusion, 
while also allowing timely correction of acute bleeding [1].

In the present case, transarterial embolization was initially performed by inserting the coils into the proximal area of the two intrahepatic pseudoaneurysms. After 10 days, rebleeding occurred in one of the intrahepatic pseudoaneurysms; although another transarterial coil embolization was planned, superselection was impossible due to a previously inserted coil and winding vascular path. However, hemobilia continued, and the biliary-intrahepatic pseudoaneurysm fistula was located through the PTBD catheter that had been inserted. After approaching the intrahepatic pseudoaneurysm through the fistula, embolization was performed using 2 Tornado coils. Review of various reports did not find any previous case of embolization of an intrahepatic pseudoaneurysm through a PTBD catheter. Hence, we report this case and a literature review.

\section{CONFLICT OF INTEREST}

No potential conflict of interest relevant to this article was reported.

\section{ORCID}

Jee Young An, https://orcid.org/0000-0002-4578-0712

Jong Ho Park, https://orcid.org/0000-0002-7275-9687

\section{REFERENCES}

1. Nagaraja R, Govindasamy M, Varma V, Yadav A, Mehta N, Kumaran V, et al. Hepatic artery pseudoaneurysms: a singlecenter experience. Ann Vasc Surg 2013;27:743-9.

2. Willson TD, Korn JM, Blecha MJ, Podbielski FJ, Connolly MM. Spontaneous rupture of a saccular intrahepatic artery aneurysm. Vasc Endovascular Surg 2012;46:679-81.

3. Baggio E, Migliara B, Lipari G, Landoni L. Treatment of six hepatic artery aneurysms. Ann Vasc Surg 2004;18:93-9.

4. Dolapci M, Ersoz S, Kama NA. Hepatic artery aneurysm. Ann Vasc Surg 2003;17:214-6.

5. Yu YH, Sohn JH, Kim TY, Jeong JY, Han DS, Jeon YC, et al. Hepatic artery pseudoaneurysm caused by acute idiopathic pancreatitis. World J Gastroenterol 2012;18:2291-4.

6. Finley DS, Hinojosa MW, Paya M, Imagawa DK. Hepatic artery pseudoaneurysm: a report of seven cases and a review of the literature. Surg Today 2005;35:543-7.

7. Tessier DJ, Fowl RJ, Stone WM, McKusick MA, Abbas MA, Sarr MG, et al. Iatrogenic hepatic artery pseudoaneurysms: an uncommon complication after hepatic, biliary, and pancreatic procedures. Ann Vasc Surg 2003;17:663-9.

8. Lumsden AB, Mattar SG, Allen RC, Bacha EA. Hepatic artery aneurysms: the management of 22 patients. J Surg Res 1996; 60:345-50.

9. Abbas MA, Fowl RJ, Stone WM, Panneton JM, Oldenburg WA, Bower TC, et al. Hepatic artery aneurysm: factors that predict complications. J Vasc Surg 2003;38:41-5.

10. Cho GH, Lee JJ, Yu SK, Kwon KA, Park DK, Kim YS, et al. A case of non-traumatic hemobilia due to pseudoaneurysm of the hepatic artery. Korean J Gastrointest Endosc 2006;33: 173-7. Korean.

11. Razik R, Fallah A, Sandroussi C, Wei AC, McGilvray ID. Dissecting pseudoaneurysm of the proper hepatic artery repaired by primary anastomosis: a case report. Case Rep Surg 2012;2012:804919. 